\title{
Influence of cultivated landscape composition on variety resistance: an assessment based on wheat leaf rust epidemics
}

\author{
Julien Papaïx ${ }^{1,2}$, Henriette Goyeau ${ }^{1}$, Philippe Du Cheyron ${ }^{3}$, Hervé Monod ${ }^{2}$ and Christian Lannou ${ }^{1}$ \\ ${ }^{1}$ INRA, UMR 1290 BIOGER, F-78850 Thiverval Grignon, France; ${ }^{2}$ INRA, UR 341 Mathématiques et Informatique Appliquées, \\ F-78350 Jouy-en-Josas, France; ${ }^{3}$ ARVALIS - Institut du végétal, F-78280 Guyancourt, France
}

Author for correspondence:

Julien Papaïx

Tel: +33134652239

Email: julien.papaix@grignon.inra.fr

Received: 8 February 2011

Accepted: 5 April 2011

New Phytologist (2011)

doi: 10.1111/j.1469-8137.2011.03764.x

Key words: Bayesian modelling, crop resistance durability, hierarchical model, landscape epidemiology, population genetics, Puccinia triticina, wheat leaf rust.

\section{Summary}

- In plant pathology, the idea of designing variety management strategies at the scale of cultivated landscapes is gaining more and more attention. This requires the identification of effects that take place at large scales on host and pathogen populations. Here, we show how the landscape varietal composition influences the resistance level (as measured in the field) of the most grown wheat varieties by altering the structure of the pathogen populations.

- For this purpose, we jointly analysed three large datasets describing the wheat leaf rust pathosystem (Puccinia triticina/Triticum aestivum) at the country scale of France with a Bayesian hierarchical model.

- We showed that among all compatible pathotypes, some were preferentially associated with a variety, that the pathotype frequencies on a variety were affected by the landscape varietal composition, and that the observed resistance level of a variety was linked to the frequency of the most aggressive pathotypes among all compatible pathotypes.

- This data exploration establishes a link between the observed resistance level of a variety and landscape composition at the national scale. It illustrates that the quantitative aspects of the host-pathogen relationship have to be considered in addition to the major resistance/virulence factors in landscape epidemiology approaches.

\section{Introduction}

In modern agriculture, structure simplification and genetic uniformity of cultivated landscapes facilitate the spread of epidemics and the genetic evolution of pathogens towards higher virulence (Oerke \& Dehne, 2004; Stukenbrock \& McDonald, 2008; Margosian et al., 2009). Nevertheless, both experimental and theoretical approaches support the idea that increasing functional diversity based on resistance factors would make agricultural systems less susceptible to diseases (Altieri, 1999; Zhu et al., 2000; Mundt, 2002; Bianchi et al., 2006; Garrett et al., 2009). More generally, a relationship between functional diversity and susceptibility to diseases had been demonstrated in ecological systems (Pautasso et al., 2005; Keesing et al., 2006) and this relationship can be considered at different spatial scales, one of them being the landscape (Gilligan, 2008). Studies in animal and human epidemiology (Keeling, 1999; Tildesley et al., 2010) have shown that landscape structure and connectivity may greatly influence pathogen invasion rates: the simplification of agricultural landscape structure and composition, along with the decline of noncrop habitat, has led to a decrease in natural pest control (Bianchi et al., 2006).

In plant pathology, the idea of designing variety management strategies at the scale of cultivated landscapes has been around for a long time (Zadoks \& Kampmeijer, 1977) and was tested more than 20 yr ago by Mundt \& Brophy (1988) with a simulation model. More recently, several authors have explored the potential of large-scale approaches to optimize the deployment of host resistance. With an approach based on the metapopulation theory (Hanski, 1998), Parnell et al. (2006) suggest that the spread of pathogen strains that are resistant to fungicides would be more effectively controlled with a landscape-scale approach, Margosian et al. (2009) 
assessed the connectivity, with regard to pathogen transmission, of the four main crops in the USA and Skelsey et al. (2009) developed a spatio-temporal model of the potato late blight pathosystem that will make it possible to evaluate spatial deployment of host resistance in large growing areas. Nevertheless, the potential of landscape epidemiology for exploring new strategies of host resistance management still remains largely underexploited by plant pathologists (Plantegenest et al., 2007). A likely reason for this is the difficulty in obtaining and analysing experimental data at a large geographic scale. A main objective of this study is therefore to contribute to filling this gap.

The development of simulation models to design variety deployment strategies in agricultural landscapes requires the identification of the effects that take place at large scales on host and pathogen populations. Most available datasets on pathogen population structure and host resistance are based on qualitative host-pathogen interactions, as described by the gene-for-gene model (Flor, 1971). It is well known that interactions between major resistance genes and avirulence genes shape pathogen population structure at large scales in cropping systems (Wolfe \& Schwarzbach, 1978; Hovmøller et al., 1993; Rouxel et al., 2003; Goyeau et al., 2006; Barrès et al., 2008) and that, reciprocally, invasions of new virulent strains render ineffective the corresponding resistance genes in the crops. Nevertheless, although the qualitative hostpathogen interactions are necessary to describe pathogen populations and to explain the observed resistance level of host varieties, they are not sufficient. Quantitative interactions determined by pathogen aggressiveness (for a review see Pariaud et al., 2009a) and host quantitative resistance (Marcel et al., 2008; Brun et al., 2010) can also play a major role in shaping pathogen populations (Miller et al., 1998; Thrall \& Burdon, 2003; Pariaud et al., 2009b). In such a case, in addition to the binary response of the gene-for-gene model, a continuous response has to be considered to describe the host-pathogen interaction.

In this paper, we test the hypothesis that landscape composition (in terms of host variety frequencies) has an impact on the changes in the observed resistance level of the main varieties grown by the farmers, at the French national scale. For this, we consider both qualitative and quantitative information on the interactions between the pathogen and its host. Data analysis focuses on a wheat (Triticum aestivum) foliar disease, leaf rust, caused by Puccinia triticina, a basidiomycete fungus (Bolton et al., 2008). This pathosystem has been studied in depth. As a result, we had access to three datasets to carry out our analysis, related to the frequencies of the most frequently grown varieties in France, a 10-yr population survey of $P$. triticina on each of these varieties and the assessment of the resistance level of these varieties in multilocal trials during the same period of time. In order to link and analyse these datasets, we developed a hierarchical model within a Bayesian framework.

\section{Description}

\section{Data description}

Wheat varieties The French institute, FranceAgriMer, publishes annual statistics on the most frequently sown wheat (Triticum aestivum L.) varieties in France (ONIGC, 2008). This dataset records the frequency, with respect to the total French wheat acreage, of the 10 most frequently sown varieties each year. From 1999 to 2008, 30 varieties were recorded, representing between $53.8 \%$ and $76 \%$ of the wheat acreage each year.

The INRA Grignon laboratory routinely identifies the major resistance genes present in the cultivated varieties. Based on this information (Goyeau et al., 2006), the recorded varieties were classified into five main groups. The first group contained the varieties bearing the resistance gene $L r 13$, and the second group the varieties with Lr14a. Groups 3,4 and 5 contained varieties with the following combinations of resistance genes: $\operatorname{Lr} 10+\operatorname{Lr} 13, \operatorname{Lr} 13+\operatorname{Lr} 37$ and $L r 10+\operatorname{Lr} 13+\operatorname{Lr} 37$, respectively. As the frequency of the first group was low and declining over the period considered, we limited our study to the last four groups.

Among these groups, we focused on the most representative varieties (in terms of frequency). Seven of them were chosen from groups 2, 3, 4 and 5: Isengrain and Soissons (group 2), Charger and Trémie (group 3), Apache (group 4) and Caphorn and Orvantis (group 5). These varieties represented between $32.2 \%$ and $60 \%$ of the French wheat acreage, depending on the year (see Supporting Information, Table S1). The other varieties, present at low frequencies, are considered together in the model as a background host population. Because only the first 10 varieties are recorded each year in the wheat survey, the frequency of those varieties in the cultivated landscape was not always known over the whole period. Apache was recorded from 2001 to 2008, Caphorn from 2003 to 2008, Charger from 1999 to 2006, Isengrain from 1999 to 2008, Orvantis from 2002 to 2008, Soissons from 1999 to 2008, and Trémie from 1999 to 2004.

Puccinia triticina population Isolates of $P$. triticina are collected each year from a network of unsprayed nurseries in 64 different locations throughout the country. A pustule is sampled at each site for each variety and the isolate is increased for pathotype determination. A detailed description of the French leaf rust survey is given in Goyeau et al. (2006). A pathotype, or phenotype for qualitative virulence (Gilmour, 1973), is attributed to each isolate collected. The pathotype indicates whether or not the isolate is able to overcome the major resistance genes.

A total of 2521 isolates were sampled on 124 varieties over the period considered (1999-2008). The varieties Soissons, Isengrain, Charger and Apache were sampled from 1999 to 2008, Trémie from 1999 to 2007, Orvantis 


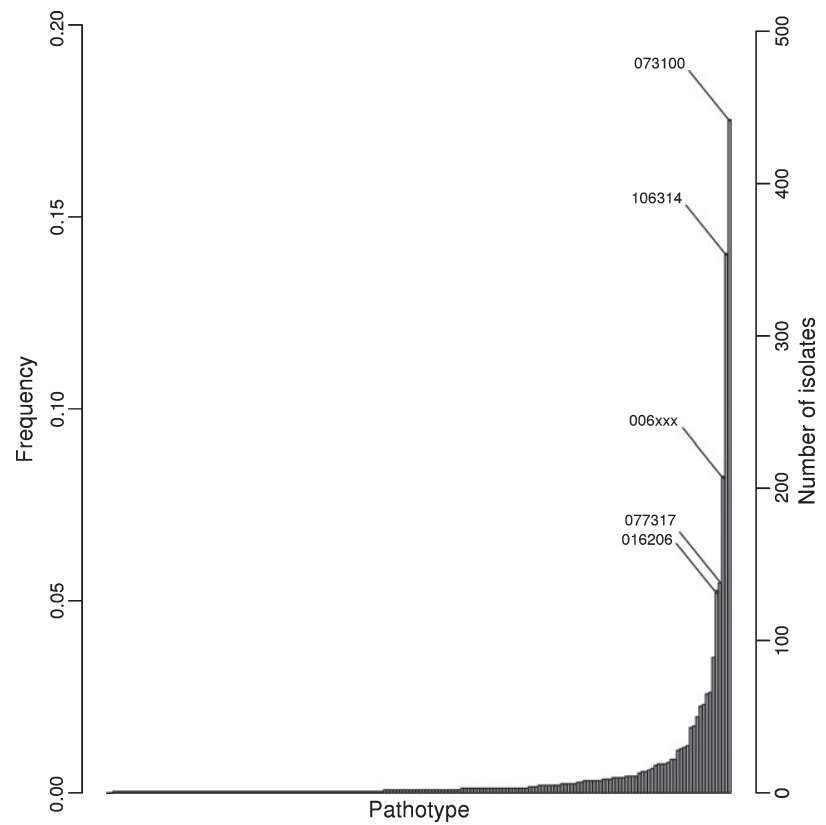

Fig. 1 Ordered frequencies and corresponding number of isolates of the pathotypes identified in the Puccinia triticina survey over the period considered (1999-2008). The pathotypes that were specifically considered in this work are indicated.

from 2001 to 2008, and Caphorn from 2003 to 2008 (Table S2). Over the whole period, 196 different pathotypes were identified, among which only a few had a high frequency (Fig. 1, Table S3). We focused on the most frequent pathotypes, referred to as 006xxx, 016206, 073100, 077317 and 106314 (see Goyeau et al., 2006). The first pathotype (006xxx) aggregates three individual pathotypes (006106, 006504 and 006506) with very close phenotypic expressions and identical microsatellite profiles. The lowfrequency pathotypes are not ignored by the model but are grouped together and considered as a background pathogen population.

Disease scoring The French technical institute, Arvalis Institut du Végétal (Guyancourt, France), carries out annual trials to evaluate the resistance level of the main wheat varieties under field conditions, in order to produce technical advice for farmers. Trials are distributed over 40 different locations throughout the wheat-growing areas in France. They consist of complete blocks containing $2 \times$ $12 \mathrm{~m}^{2}$ plots (10 rows) sown with several varieties. The sowing date is chosen as an average of the optimal sowing dates of all the varieties. Sowing density and nitrogen fertilization are determined according to local practices, based on soil type, expected yield, etc. The plots are not sprayed with pesticides. The variety resistance level is directly evaluated from the disease scoring as observed resistance level $=1-$ disease score, where the disease score is the proportion of diseased leaf surface. In this paper, 'observed resistance' is defined as the resistance level of a variety as it is scored in the field (the term 'field resistance' is sometimes used in the literature) and represents the variety resistance as it is perceived by farmers and advisors. This 'observed resistance' is the result of environmental conditions, the genetic resistance factors of the varieties and the genetic composition of the pathogen population.

Scoring of the leaf rust symptoms is performed once a year in May or June. The Apache variety was scored from 1999 to 2008, Caphorn from 2001 to 2008, Charger from 1999 to 2007, Isengrain from 1999 to 2005, Orvantis from 2000 to 2008, Soissons from 1999 to 2008, and Trémie from 1999 to 2004 (Fig. 2).

Disease scoring yields both qualitative information (presence/absence of disease, usually referred to as 'incidence' in

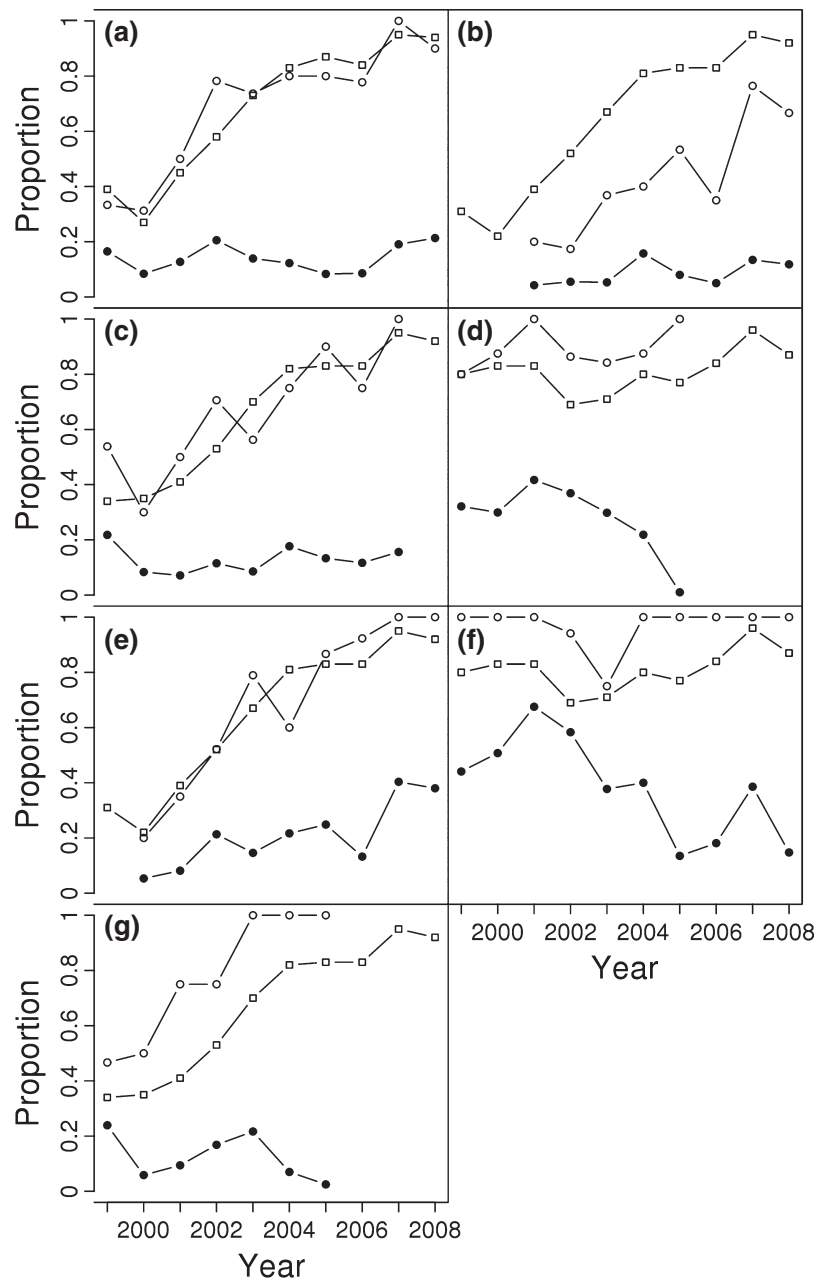

Fig. 2 Evolution across all observation sites of the Arvalis survey of leaf rust incidence (open circles) and disease scores (closed circles), along with the frequency of pathotypes (open squares) that are virulent for wheat (Triticum aestivum L.) varieties Apache (a), Caphorn (b), Charger (c), Isengrain (d), Orvantis (e), Soissons (f), and Trémie (g). The relationship between disease scores and time was tested by a GLM with beta distributed errors. The slope was significantly $>0$ for Orvantis and $<0$ for Soissons, with a 0.001 threshold. 
the phytopathological literature) and quantitative information (level of observed disease, usually referred to as 'severity' - Fig. 2). Absence of disease results either from the absence of the pathogen itself or, more likely in the case of leaf rust, from incompatibility of the pathotypes that were present at the scoring time with the variety considered. As classically observed, disease incidence on a variety was significantly linked to the proportion of virulent pathotypes in the pathogen population (Fig. 2). In order to account for such effects, qualitative virulence is introduced in the statistical model but, as stated before, we focus on the quantitative aspects of the host-pathogen relationship when exploring the datasets.

\section{Statistical modelling}

We constructed a statistical model in order to jointly analyse the three large-scale datasets describing the wheat leaf rust pathosystem. The model aims to look for the existing correlations between the wheat variety frequencies and the $P$. triticina population composition, and between the $P$. triticina population composition and the observed disease severity on the main wheat varieties. The need for a convenient and flexible framework to combine information from several parallel data sources led us to develop a statespace model (SSM). In a SSM, the datasets are first described by observation variables that constitute the observation process layer (Fig. 3). The observation variables are then linked to each other via unobserved hidden variables, classically referred to as 'latent variables' that constitute the system process layer. The model thus consisted of two sets of equations. The state equations described the statistical links between the latent variables. The observation equations linked the latent variables and the observation variables. Parameters appear both in the system and observation process layers.

With regard to the biological system, the model was broken down into two submodels (Fig. 3). The population composition submodel was centred on the $P$. triticina population composition and on its dependence on wheat variety frequencies. The disease severity submodel was dedicated to the influence of $P$. triticina population composition on the observed leaf rust severity for each wheat variety.

We first present the main characteristics and assumptions of the model. We then describe the population composition submodel and the disease severity submodel, how they are

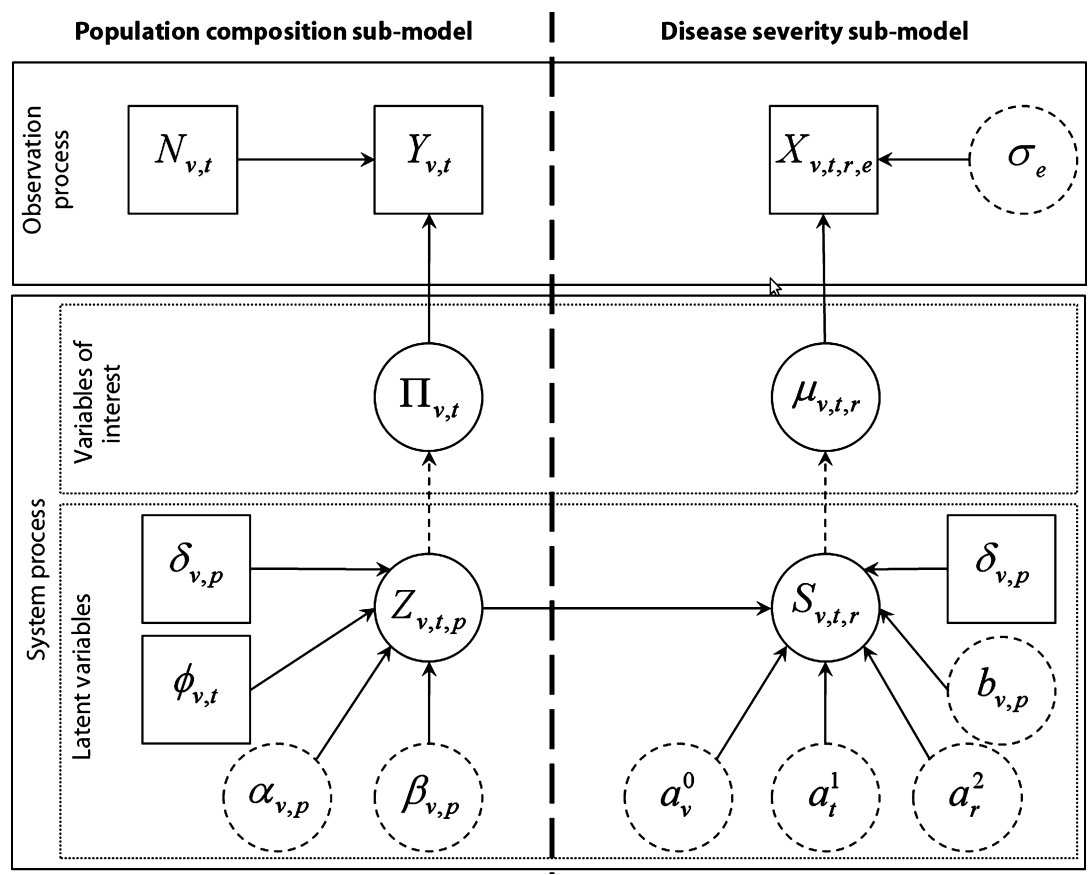

Fig. 3 Graphical representation of the state-space model ( $p$, pathotype; $v$, variety; $t$, year; $r$, climatic region; $e$, trial in Arvalis survey). The system process is composed of the latent variables (solid circles), $Z_{v, t, p}$ and $S_{v, t, r}$ depending on unknown parameters (dashed circles) and covariables (solid squares) via stochastic links (solid arrows). From these latent variables, the unobserved variables of interest (solid circles), $\Pi_{v, t}$ (the vector of pathotype proportions on variety $v$, year $t$ ) and $\mu_{v, t, r}$ (the mean proportion of diseased leaf area of variety $v$, year $t$ in region $r$ ), are deduced via a deterministic link (dashed arrows). The observed data (solid square) are the visible part of the system process. In the population composition submodel, $Y_{v, t}$ classifies the leaf rust pustules sampled on variety $v$, year $t$, for the $P$ pathotypes. In the disease severity submodel, $X_{v, t, r, e}$ denote the disease score attributed to variety $v$ on trial $e$, year $t$ and region $r$ in the Arvalis survey. The parameter $\sigma_{e}$ (dashed circle) represents the within-trial variability. The other parameters are: $\phi_{v, t}$ (variety frequencies), $\delta_{v, p}$ (variety-pathotype compatibility), $\alpha_{v, p}$ (basic affinity), $\beta_{v, p}$ (sensitivity to changes in variety frequencies), $a_{v}^{0}$ (variety effect), $a_{t}^{1}$ (year effect), $a_{r}^{2}$ (region effect) and $b_{v, p}$ (sensitivity to changes in pathotype frequencies). 
New

Phytologist

Table 1 Definitions of the main terms and parameters used in this study

\begin{tabular}{|c|c|c|}
\hline Terms & Symbols & Description/biological interpretation \\
\hline Pathotype & $p$ & Phenotype for qualitative virulence \\
\hline Disease score & - & Proportion of diseased leaf surface \\
\hline Observed resistance level & - & $\begin{array}{l}1 \text { - disease score (results from genetic resistance factors of the varieties, } \\
\text { genetic composition of the pathogen population and environmental conditions) }\end{array}$ \\
\hline Incidence & - & Presence/absence of disease (qualitative information resulting from the disease scores) \\
\hline Severity & - & Level of observed disease (quantitative information resulting from the disease scores) \\
\hline Aggressiveness & - & Quantitative component of pathogenicity \\
\hline Virulence & $\delta$ & Capacity of a pathotype to overcome a major resistance gene \\
\hline Basic affinity & $\alpha$ & Indication of a pathotype aggressiveness on a variety at the landscape scale \\
\hline- & $\beta$ & Response of the pathogen to changes in the landscape composition \\
\hline- & $a^{0}$ & Susceptibility of a variety confronted to the global leaf rust population \\
\hline- & $a^{1}$ & Year effect \\
\hline- & $a^{2}$ & Climatic region effect \\
\hline- & $b$ & Effect of the proportion of a pathotype on the observed disease on a variety \\
\hline- & $C_{1}$ & $\begin{array}{l}\text { For a given variety, minimal value of the posterior probability that a particular parameter } \\
\text { for a pathotype is greater than the same parameter for another pathotype }\end{array}$ \\
\hline- & $C_{2}$ & $\begin{array}{l}\text { For a given pathotype, minimal value of the posterior probability that a particular parameter } \\
\text { for a variety is greater than the same parameter for another variety }\end{array}$ \\
\hline
\end{tabular}

connected and how the parameters can be interpreted in biological terms. Finally, we define the criteria that were used to interpret the results. Additional technical information is given in Notes $\mathrm{S} 1$ and Table 1 provides a summary of terms and parameters definitions.

Model characteristics and assumptions The host landscape was considered as a set of $V=7$ varieties with yeardependent frequencies and a background landscape with low but unknown variety frequencies. The pathogen population was composed of $P=5+1$ pathotypes: the five individual pathotypes presented in the Data Description section, plus a generic pathotype that included all the other pathotypes (see Fig. 1). The pathotype distribution was assumed to be homogeneous at the scale of the country (Goyeau et al., 2006). No explicit dependence from one year to the next one was included (i.e. there is no time dependence in the model). As data were collected at the end of the epidemic season, the effect of the current year on the pathogen population structure was assumed to be predominant over the potential effect of previous years. Although the analysis was done at the French national scale, we considered $r=7$ climatic regions (southeast, southwest, centresouth, centre, northeast, north and northwest of France) in order to account for the influence of climatic conditions on disease development in the disease severity submodel.

Population composition submodel In the leaf rust survey, year $t, N_{v, t}$ pustules were sampled on variety $v$ and each one was classified as one of the $P$ pathotypes defined above. Let $Y_{v, t}=\left(y_{v, t, 1}, \cdots, y_{v, t, P}\right)$ be the categorical random variable whose elements $y_{v, t, p}$ denote the number of leaf rust pustules sampled on variety $v$, year $t$, and assigned to pathotype $p$. Let $\pi_{v, t, p}$ denote the proportions of pathotype $p$ on variety $v$, year $t$. Assuming that the probability for pathotype $p$ to be sampled only depends on its proportion, $Y_{v, t}$ has a multinomial distribution with parameters $N_{v, t}$ and $\Pi_{v, t}=\left(\pi_{v, t, 1}, \cdots, \pi_{v, t, P}\right)$. This led to the observation equation that describes the $P$. triticina population composition:

$Y_{v, t} \mid N, \Pi_{v, t} \sim \operatorname{Multinomial}\left(N_{v, t}, \Pi_{v, t}\right)$.

In the state part of this submodel (Fig. 3), the proportions $\pi_{v, t, p}$ were associated with a latent variable $Z_{v, t, p}$ that represents the relative population size of pathotype $p$ on variety $v$ year $t$. Note that $Z_{v, t, p}$ is not the actual size of the pathotype population but a scale variable instead. $\pi_{v, t, p}$ and $Z_{v, t, p}$ were linked through the state equations (see Notes S1 for more details):

$\left\{\begin{array}{l}E\left[Z_{v, t, p}\right]=\bar{Z}_{v, t, p} \\ =\alpha_{v, p}+\sum_{i=1}^{V} \delta_{i, p} \cdot \beta_{i, p} \cdot \phi_{i, t},\left(\alpha_{v, p}, \beta_{v, p}\right) \geq 0, \\ Z_{v, t, p} \mid \bar{Z}_{v, t, p} \sim \operatorname{Gamma}\left(\bar{Z}_{v, t, p}, 1\right), \\ \pi_{v, t, p}=\frac{Z_{v, t, p}}{Z_{v, t, 1}+\ldots+Z_{v, t, p}} .\end{array}\right.$

Eqn 1

In Eqn 1(a), $\alpha_{v, p}$ represents a basic affinity between pathotype $p$ and variety $v$, and the second term accounts for the effect of the landscape composition, variable $\phi_{\nu, t}$ denoting the frequency of variety $v$ during year $t$, and parameter $\beta_{v, p}$, denoting the sensitivity of pathotype $p$ to the frequency of variety $v$. Previous information on the gene-for-gene relationship was integrated through the binary parameter $\delta_{v, p}$ defined by: 
$\delta_{v, p}= \begin{cases}1 & \text { if } p \text { and } v \text { are compatible } \\ 0 & \text { otherwise. }\end{cases}$

Note that in Eqn 1(a), there can be a nonzero basic affinity even if the variety and the pathotype are incompatible $\left(\delta_{v, p}=0\right)$. This is to take the situation in which an incompatible pathotype is nonetheless able to produce a few pustules on a resistant variety into account, as sometimes occurs in field epidemics (Samborski, 1985).

In this statistical model, the links that were established between each component are descriptive and the associated coefficients should not be directly assimilated to biological parameters of the plant-pathogen relationship. Eqn 1(a) can, however, be interpreted as follows. The basic affinity $\alpha_{v, p}$ of pathotype $p$ for variety $v$ is the part of $E[Z]$ that is not accounted for by the frequencies in the landscape of the seven varieties considered in the analysis. A high $\alpha_{\nu, p}$ value means that pathotype $p$ was always well represented on variety $v$ during the period studied, regardless of the landscape composition. Therefore, for a compatible pathotype, this parameter provides an indication of pathotype aggressiveness on a variety, based on the size of its population on that variety and relative to the other pathotypes. Parameter $\beta$ quantifies the response of the pathogen to changes in the landscape composition. A simple analogy can be made here with a linear regression, with $\alpha$ being analogous to the intercept and $\beta$ to the slope.

Disease severity submodel Let $X_{v, t, r, e}$ denote the disease score attributed in the Arvalis survey to variety $v$ for trial $e$, year $t$ and in region $r$. Its expectation $\mu_{v, t, r}$ was assumed to reflect the mean rust severity on variety $v$ that year in that region. The score $X_{v, t, r, e}$ varied in the $[0,1]$ interval but the actual range of notation differed between trials. Consequently, the scores were assumed to follow a beta distribution with mean value, $\mu_{v, t, r}$ and scale parameter $\sigma_{e}$ depending on the trial. This led to the observation equation that describes the disease scoring dataset:

$X_{v, t, r, e} \mid \mu_{v, t, r}, \sigma_{e} \sim \operatorname{Beta}\left(\mu_{v, t, r}, \sigma_{e}\right)$.

The expected disease scores $\mu_{v, t, r}$ were associated with two latent variables $S_{v, t, r}$ and $S_{v, t, r}$ through the state equations (see Notes $S 1$ for more details):

$$
\left\{\begin{array}{l}
E\left[S_{v, t, r}\right]=\bar{S}_{v, t, r}= \\
a_{v}^{0}+a_{t}^{1}+a_{r}^{2}+\sum_{j=1}^{P-1} \delta_{v, j} \cdot b_{v, j} \cdot \pi_{v, t, j}, \quad\left(a_{v}^{0}, a_{t}^{1}, a_{r}^{2}, b_{v, p}\right) \geq 0, \\
S_{v, t, r} \mid \bar{S}_{v, t, r} \sim \operatorname{Gamma}\left(\bar{S}_{v, t, r}, 1\right), \\
\mu_{v, t, r}=\frac{S_{v, t, r}}{S_{v, t, r}+S_{v, t, r}^{\prime}} .
\end{array}\right.
$$

Eqn 2
As disease scores are defined in the scoring procedure as the observed proportion of diseased leaf area, $\mu_{v, t, r}=\mathrm{E}\left[X_{v, t, r, e}\right]$ can be identified as the mean proportion of leaf area that was diseased for variety $v$ during year $t$ in region $r$. In Eqn 2, $S_{v, t, r}$ represents the diseased leaf area of a variety $v$, year $t$, in region $r$, and $S_{v, t, r}$ denotes the healthy leaf area. As for $Z, S$ and $S$ should not be considered as actual areas but as scale variables instead. In Eqn 2(a), parameters $a^{0}, a^{1}$ and $a^{2}$ represent the variety, year and region main effects, respectively, and define a basal disease pressure. In particular, $a^{0}$ can be seen as the basic susceptibility of a variety confronted with the global leaf rust population over the entire period considered. The last term of the equation accounts for the effect of the pathogen population composition, where $\delta$ still denotes $0-1$ qualitative virulence. Here again, $b$ would be analogous to a slope, quantifying the link between the proportion of a particular pathotype and the observed disease on a variety, whereas $a^{0}+a^{1}+a^{2}$ could be assimilated to an intercept. The summation in Eqn 2(a) is performed over the $(P-1)$ individual pathotypes considered earlier. The index $P$ identifies the generic pathotype that groups all the other pathotypes that were present during the period. By construction, their joint effect is included in the basal disease pressure.

Index and criterion definitions In the model defined earlier, the interactions between pathotypes and varieties are quantified through the parameters $\theta_{v, p}$, with $\theta$ in $\{\alpha, \beta, b\}$. In order to synthesize the resulting information, two sets of criteria varying in $[0,1]$ were defined:

$C_{1}(\theta ; v, p)=\min _{p^{\prime}}\left\{\operatorname{Prob}\left(\theta_{v, p}>\theta_{v, p^{\prime}}\right)\right\}$

$C_{2}(\theta ; v, p)=\min _{v^{\prime}}\left\{\operatorname{Prob}\left(\theta_{v, p}>\theta_{v^{\prime}, p}\right)\right\}$,

where $\operatorname{Prob}(\cdot)$ denotes posterior probabilities. In the first case $\left(C_{1}(\theta ; v, p)\right)$, the variety $v$ is fixed and $\theta_{v, p}$ values are compared among pathotypes. In the second case $\left(C_{2}(\theta ; v, p)\right)$, the pathotype $p$ is fixed and $\theta_{v, p}$ values are compared among varieties. With these definitions, large $C_{1}(\theta ; v, p)$ values identify the pathotypes that are most strongly linked to variety $v$ with respect to parameter $\theta$, whereas, in a symmetrical way, large $C_{2}(\theta ; v, p)$ values identify the varieties to which pathotype $p$ is the most strongly linked with respect to parameter $\theta$.

In order to understand how the pathogen population responds to host frequencies in the cultivated landscape, it is worthwhile comparing the effect of the basic affinity and the effect of the landscape composition, as defined in Eqn 1(a). The relative weights of both types of effects were defined by: 
New

Phytologist

$$
W(\alpha ; v, t, p)=\frac{\alpha_{v, p}}{\alpha_{v, p}+\sum_{i=1}^{V} \delta_{i, p} \cdot \beta_{i, p} \cdot \phi_{i, t}}
$$

and

$$
W(\beta, i ; v, t, p)=\frac{\delta_{i, p} \cdot \beta_{i, p} \cdot \phi_{i, t}}{\alpha_{v, p}+\sum_{i=1}^{V} \delta_{i, p} \cdot \beta_{i, p} \cdot \phi_{i, t}} .
$$

\section{Bayesian implementation}

Inference on the parameters was performed by Bayesian statistical methods, resulting in a joint posterior distribution (Gelman et al., 2004). This posterior distribution was computed via a Markov Chain Monte Carlo (MCMC) method using JAGS software (Plummer, 2010).

Prior densities Only the $\delta$ parameters were considered to be known a priori. For all the other parameters, no a priori information was assumed to be available and noninformative prior densities were used: $\alpha, \beta, a^{0}, a^{1}, a^{2}$ and $b$ were defined as being uniformly distributed on $[0,10000]$ and the trial variances $\sigma$ were defined as being uniformly distributed on $[0,1]$. It was systematically verified that the upper bound of the uniform prior density was large enough to have no influence on the posterior densities.

For identification reasons, we fixed $\bar{Z}_{v, t, P}=1, \forall(v, t)$ and $S_{v, t, r}=5, \forall(v, t, r)$. This choice was made after checking the data-fitting and performing sensitivity analyses. In particular, it was verified that variation around the chosen values had little impact on the criteria defined above.

MCMC convergence and mixing Three MCMC-chains of 125000 iterations were computed. Convergence was assessed using the Gelman and Rubin statistic $(\hat{R})$ which compares the within to the between variability of chains started at different and dispersed initial values (Gelman et al., 2004). Burn-in was set to 25000 , and thinning every 100 iterations resulted in acceptable mixing and convergence $(\hat{R}<1.1$ for all the parameters).

Data fitting To assess the fit of the model to the data, we used an approach known as posterior predictive checking, which is a Bayesian counterpart of the classical tests for goodness-of-fit (Gelman et al., 2004). The idea is to generate replicated data from the posterior distribution of the parameters. If the model fits the data well, then the replicated data should be similar to the observed data. For both pathotype proportion and disease score, the posterior distributions of the replicated data showed an adequate fit of the model (Fig. 4).
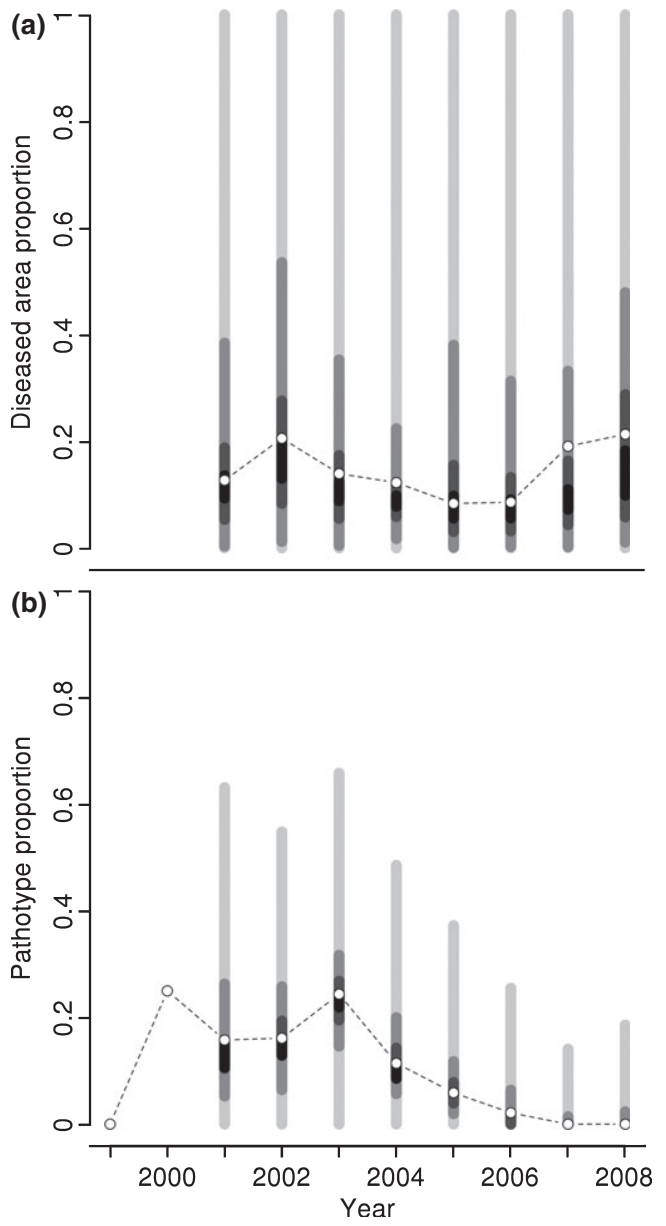

Fig. 4 Posterior densities of replicated data (tinted bars), generated from the posterior distribution of the parameters, and observed data (open circles). Example of the mean disease score on wheat (Triticum aestivum L.) variety Apache (a) and the proportion of pathotype 016206 (b). Tinted bars are median-centred quantile intervals, from the darkest to the lightest: $(0.45,0.55),(0.35,0.65)$, $(0.15,0.85)$ and $(0,1)$. The model does not incorporate timedependency between years.

\section{Results}

\section{Overview}

In a first step, the model is used to explore the relationship between the host and the pathogen populations. Two aspects are considered: the basic affinity between pathotypes and varieties, described by parameter $\alpha$, and the response of the pathogen to changes in the landscape composition, described by parameter $\beta$. Posterior densities of parameters $\alpha$ and $\beta$ are given in Figs $S 1, S 2$ but more synthetic information is provided by criterion $C_{1}(\theta ; v, p)$ and $C_{2}(\theta ; v, p)$, with $\theta=\alpha$ or $\beta$, which will be used to discuss how the pathogen population responds to changes in the host population (Tables 2,3).

In a second step, we attempt to link the observed resistance in the field to the composition of the pathogen population. Criterion $C_{1}(b ; v, p)$ (Table 2) is used to 
describe how the disease level observed on a variety can be linked to the pathogen population composition. Posterior densities of the parameters are given in Fig. S3.

\section{Variety frequencies and pathogen population composition}

Basic affinity $(\boldsymbol{\alpha})$ The $C_{1}$ criterion (Table 2) indicates the dominance of a pathotype on a variety. Pathotypes 106314 and 006xxx were dominant on the two most recent varieties, Caphorn and Orvantis (released in $2000)$, respectively $\left(C_{1}(\alpha ;\right.$ Caphorn, 106314$)=0.81$ and $C_{1}(\alpha ;$ Orvantis,006xxx $\left.)=0.78\right)$. These two pathotypes are also the most recent ones in the French $P$. triticina population (no isolates of these pathotypes were found before 2003 and 2002, respectively). Pathotype 073100 was clearly dominant on varieties Isengrain and Soissons. Two pathotypes, 016206 and 073100, were dominant on Trémie. Pathotype 016206 was moderately dominant on Charger. No pathotype was found to be specifically dominant on
Apache. Note that the Trémie-073100 association was unexpected here as, according to its qualitative virulence pattern, this interaction should be incompatible (Goyeau et al., 2006). Nevertheless, infection of Trémie with that pathotype often produces a few viable pustules. As the frequency of 073100 in the pathogen population was high and the disease levels (total number of pustules) on Trémie were low, it was logical to detect a link between Trémie and 073100 .

Criterion $C_{2}$ (Table 3) makes it possible to determine the preference of a pathotype for one or several of the seven varieties considered. Pathotype 006xxx was found preferentially on Orvantis but was also present on Apache and Caphorn. These three varieties are those that share the Lr37 resistance gene. By contrast, pathotype 106314 was only related to Caphorn $\left(C_{2}(\alpha ;\right.$ Caphorn,106314 $\left.)=0.94\right)$. Pathotype 073100 was highly abundant on Soissons and Isengrain and was never related to the other varieties $\left(C_{2}=0\right)$. Pathotype 016206 was related to Trémie and was infrequently found on Isengrain and Soissons. Finally, path-

Table 2 Values of the $C_{1}$ criterion for each parameter and each pathotype-variety pair

\begin{tabular}{|c|c|c|c|c|c|c|c|c|c|c|c|c|c|c|c|c|c|c|c|c|c|}
\hline & \multicolumn{3}{|c|}{ Apache } & \multicolumn{3}{|c|}{ Caphorn } & \multicolumn{3}{|c|}{ Charger } & \multicolumn{3}{|c|}{ Isengrain } & \multicolumn{3}{|c|}{ Orvantis } & \multicolumn{3}{|c|}{ Soissons } & \multicolumn{3}{|c|}{ Trémie } \\
\hline & $\alpha$ & $\beta$ & $b$ & $\alpha$ & $\beta$ & $b$ & $\alpha$ & $\beta$ & $b$ & $\alpha$ & $\beta$ & $b$ & $\alpha$ & $\beta$ & $b$ & $\alpha$ & $\beta$ & $b$ & $\alpha$ & $\beta$ & $b$ \\
\hline $06 x x x$ & 0.40 & 0.21 & 0.36 & 0.19 & 0.29 & 0.32 & 0.23 & 0.16 & 0.53 & 0.00 & 0.11 & - & 0.78 & 0.26 & 0.20 & 0.00 & 0.14 & 0.04 & 0.03 & 0.18 & - \\
\hline 016206 & 0.45 & 0.38 & 0.55 & 0.15 & 0.00 & 0.28 & 0.64 & 0.57 & 0.47 & 0.00 & - & - & 0.22 & 0.40 & 0.07 & 0.00 & - & - & 0.51 & 0.59 & - \\
\hline 073100 & 0.55 & - & - & 0.09 & - & - & 0.24 & - & - & 1.00 & 0.73 & - & 0.10 & - & - & 1.00 & 0.70 & 5 & 0.49 & - & - \\
\hline 077317 & 0.37 & 0.62 & 0.45 & 0.06 & 0.00 & 0.68 & 0.36 & 0.43 & 0.45 & 0.00 & 0.27 & - & 0.15 & 0.60 & 0.16 & 0.00 & 0.30 & 0.05 & 0.08 & 0.41 & - \\
\hline 106314 & 0.25 & 0.13 & 0.31 & 0.81 & 0.71 & 0.10 & 0.28 & 0.12 & 0.38 & 0.00 & 0.09 & - & 0.08 & 0.16 & 0.80 & 0.00 & 0.11 & 0.00 & 0.03 & 0.16 & - \\
\hline
\end{tabular}

This criterion compares the parameter values among pathotypes for a given variety. Large $C_{1}(\theta ; v, p)$ (with $\theta$ in $\{\alpha, \beta, b\}$ ) values identify the pathotypes that are most strongly linked to variety $v$ according to parameter $\theta$.

$\alpha$, basic affinity of a pathotype for a variety.

$\beta$, sensitivity of pathotypes to changes in variety frequencies.

$b$, sensitivity of disease severity on a variety to the pathotype proportions.

Table 3 Values of the $C_{2}$ criterion for parameters $\alpha$ and $\beta$ for each pathotype-variety pair

\begin{tabular}{|c|c|c|c|c|c|c|c|c|c|c|}
\hline & \multicolumn{2}{|c|}{$006 x x x$} & \multicolumn{2}{|c|}{016206} & \multicolumn{2}{|c|}{073100} & \multicolumn{2}{|c|}{077317} & \multicolumn{2}{|c|}{106314} \\
\hline & $\alpha$ & $\beta$ & $\alpha$ & $\beta$ & $\alpha$ & $\beta$ & $\alpha$ & $\beta$ & $\alpha$ & $\beta$ \\
\hline Apache & 0.17 & 0.00 & 0.19 & 0.19 & 0.00 & - & 0.44 & 0.20 & 0.06 & 0.00 \\
\hline Caphorn & 0.35 & 0.95 & 0.29 & 0.14 & 0.00 & - & 0.37 & 0.15 & 0.94 & 1.00 \\
\hline Charger & 0.09 & 0.00 & 0.25 & 0.35 & 0.00 & - & 0.40 & 0.20 & 0.06 & 0.00 \\
\hline Isengrain & 0.05 & 0.00 & 0.11 & - & 0.62 & 0.52 & 0.56 & 0.11 & 0.03 & 0.00 \\
\hline Orvantis & 0.65 & 0.05 & 0.23 & 0.64 & 0.00 & - & 0.44 & 0.21 & 0.03 & 0.00 \\
\hline Soissons & 0.04 & 0.00 & 0.01 & - & 0.38 & 0.48 & 0.44 & 0.13 & 0.02 & 0.00 \\
\hline Trémie & 0.05 & 0.00 & 0.71 & 0.36 & 0.00 & - & 0.33 & 0.21 & 0.02 & 0.00 \\
\hline
\end{tabular}

This criterion compares the parameter values among varieties for a given pathotype. Large values of $C_{2}(\theta ; v, p)$ (with $\theta$ in $\left.\{\alpha, \beta\}\right)$ identify the varieties to which pathotype $p$ is the most strongly linked according to parameter $\theta$.

$\alpha$, basic affinity of a pathotype for a variety.

$\beta$, sensitivity of pathotypes to changes in variety frequencies. 
otype 077317 was identified as a generalist, with no preference for any of the varieties.

Response of pathogen to changes in landscape composition $(\boldsymbol{\beta})$ Changes in the frequencies of Isengrain and Soissons in the cultivated landscape strongly affected the proportion of pathotype 073100 in the pathogen population (Table 2). Changes in the frequencies of Apache, Charger, Orvantis and Trémie mainly affected 016206 and 077317 (Table 2, Fig. S2), but 016206 was more especially affected by Orvantis (Table 3). As for $\alpha$, pathotype 077317 was not specifically sensitive to any of these varieties (Table 3). Changes in the frequency of Caphorn mainly affected pathotypes 006xxx and 106314 (Table 2, Fig. S2) and, among the varieties considered, Caphorn was the most influential variety on both pathotypes (Table 3). Data analysis also suggested that changes in Orvantis influenced pathotype 006xxx (Fig. S2). Note that although pathotype 006xxx presented a strong affinity $\alpha$ for Orvantis, it did not appear very sensitive to changes in the proportion of Orvantis in the host population (Table 3, parameter $\beta$ ). A simple reason for that is that Orvantis's frequency did not vary much between 2002 and 2008 (Table S1).

Relative effect of basic affinity and changes in variety frequencies ( $W$ ) Fig. 5 shows the respective weights of the basic affinity $W(\alpha ; v, t, p)$ and of the changes in the variety frequencies $W(\beta, i ; v, t, p)$ on the population size of a pathotype on a variety, as estimated by $Z$ (Eqn 1$)$. Overall, Fig. 5 suggests that the frequency of a pathotype on a variety is the highest when the landscape composition is the most favourable, that is, when $W(\alpha ; v, t, p)$ is minimal.

The presence of 073100 on Soissons was mainly explained by its very high affinity for this variety (Fig. 5a). Conversely, the frequency of 106314 on the same variety was mainly related to the frequency of Caphorn in the landscape (Fig. 5c). The case of 077317 is more complex. Changes in the landscape composition influenced this pathotype through a combination of varieties that varied over time: mainly Trémie and Soissons in the beginning of the period, then Charger and finally Apache and Orvantis (Fig. 5b).

In the right column of Fig. 5, we examine the situation of pathotype 106314. The frequency of this pathotype on Caphorn was explained by both its affinity $(\alpha)$ and the increasing frequency of Caphorn in the landscape, with weights of comparable magnitude (Fig. 5e). This suggests an amplification effect, with the increase in Caphorn in the landscape (Table S1) resulting in a higher frequency of 106314 on this variety. On Soissons and Apache (Figs 5c,d), 106314 increased from 0\% in 2003 to 35\% and $39 \%$, respectively, in 2008, and this increase was accounted for by the frequency of Caphorn in the land-

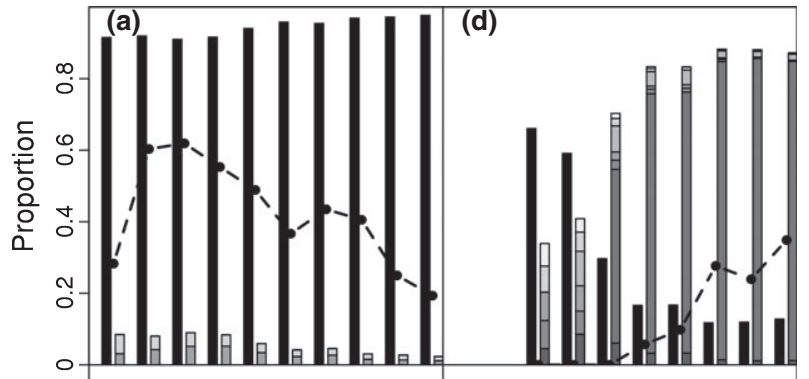

(b)

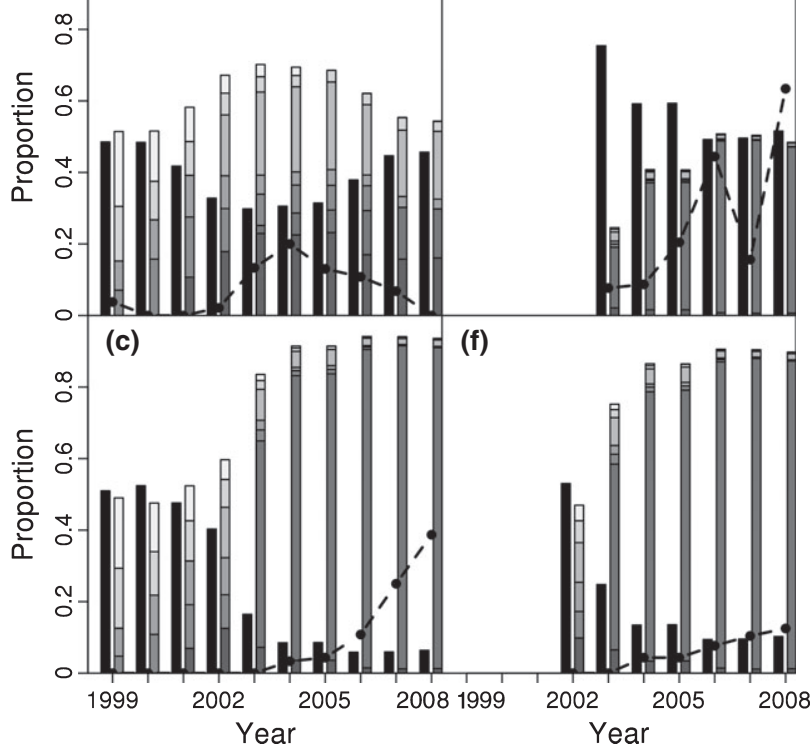

Fig. 5 Observed proportion of pathotype $p$ on variety $v$ (closed circles) along with associated model-based indices (bars), for selected ( $p, v)$ pairs. Pathotypes 073100 (a), $077317(b)$ and 106314 (c) on wheat (Triticum aestivum L.) variety Soissons; pathotype 106314 on varieties Apache (d), Caphorn (e) and Orvantis (f). Weights of basic affinity, $W(\alpha ; v, t, p)$ : closed bars and changes in landscape composition, $\mathrm{W}(\beta, i ; v, t, p)$ : tinted bars, on the considered pathotype frequency. Tinted bars indicate varieties Apache, Caphorn, Charger, Isengrain, Orvantis, Soissons and Trémie from the darkest to the lightest.

scape. On Orvantis, pathotype 106314 also increased in frequency, probably for the same reasons, but it only reached $13 \%$ of the pathogen population sampled on this variety at the end of the period (Fig. 5f). This might be because of competition with 006xxx, which had a high affinity for Orvantis with respect to the other pathotypes (Table 2).

\section{Disease dynamics}

Effect of pathogen population composition on observed resistance $(b)$ For Isengrain and Trémie, very large credibility intervals were obtained for parameter $b$ (Fig. S3), probably because pathotypes 006xxx and 106314 were observed for only 1 or $2 \mathrm{yr}$ on these varieties, which made the data difficult to exploit. On the other varieties, three 
different kinds of responses were obtained. For Apache, Caphorn and Charger, the disease scorings yielded constant values (Fig. 2) and it seems difficult to link the observed resistance levels to a specific pathotype. Nevertheless, the disease scores on Apache could be related to the proportions of pathotypes 077317 and 016206 , and the disease scores on Caphorn could be related to 077317 (Table 2, Fig. S3). It is interesting to note that the $b$ value for the pair Caphorn-106314 was very low, even though the frequency of 106314 was strongly linked to Caphorn (Tables 2, 3). This suggests that Caphorn, when grown extensively, influenced the pathotype frequencies in the pathogen population without being affected by severe epidemics itself. The second kind of response is that of Orvantis, for which the observed resistance level was decreasing (so that the disease scores kept increasing - Fig. 2). This was correlated to the increase in 106314 in the pathogen population $\left(C_{1}(b\right.$; Orvantis, 106314$\left.)=0.80\right)$. The last response type is that of Soissons, on which the observed resistance increased (and disease scores decreased - Fig. 2). This could be linked to the decrease in the frequency of 073100 in the pathogen population $\left(C_{1}(b ;\right.$ Soissons, 073100$\left.)=0.95\right)$.

Variety, year and region effects The variety effect, $a^{0}$, can be used as a criterion to rank the varieties according to their susceptibility to leaf rust, taking both qualitative and quantitative pathogenicity into account. As expected, Isengrain and Soissons had the highest $a^{0}$ values and Caphorn the lowest (Fig. S4a). The year effect, $a^{1}$ (Fig. S4b), accounted for the P. triticina population breakdown in 2003 and subsequent changes in the following years (Goyeau et al., 2006). The region effect, $a^{2}$ (Fig. S4c), was consistent with the known behaviour of the pathogen, notably that northern and south-eastern France are not favourable to wheat leaf rust.

\section{Discussion}

In this article, we developed a statistical model in order to jointly analyse three large-scale datasets describing the wheat leaf rust pathosystem. Many published papers establish a relationship between the frequency of resistance genes in the host population and the evolution of the pathogen population structure in terms of pathotypes, based on qualitative virulence factors (Hovmøller et al., 1993; Kolmer, 2002; Goyeau et al., 2006). The originality of the present approach was to account for the quantitative aspects of the host-pathogen relationship and to relate host and pathogen genotype frequencies to observed disease severity values. The analysis demonstrated that the landscape varietal composition influences the observed resistance level of the most frequently grown wheat varieties by altering the structure of the pathogen population. Another conclusion of the study is that quantitative effects (linked either to host quantitative resistance or pathogen aggressiveness) played a major role in shaping the leaf rust population structure in France over the past $10 \mathrm{yr}$.

The analysis of the link between the pathogen population composition (in terms of pathotypes) and the landscape varietal composition revealed preferential associations between varieties and pathotypes that were not accounted for by the known compatibility relationships based on avirulenceresistance interactions. The strength of these associations is quantified in the model by parameter $\alpha_{\nu, p}$ (see Eqn 1a), which can be interpreted as an indication of the aggressiveness level of a pathotype on a variety. Two compatible (virulent) pathotypes may have very different $\alpha$ values on a variety, as in the case of $073100\left(\alpha_{\text {Soissons, } 073100}=2.2\right.$, $\left.I C_{95 \%}=[1.2,3.5]\right)$ and 106314 ( $\alpha_{\text {Soissons, } 106314}=0.057$, $\left.I C_{95 \%}=[0.0023,0.30]\right)$ on Soissons. This means that although 106314 is fully compatible with Soissons according to the gene-for-gene relationship, it exhibits a low aggressiveness on Soissons in the field, whereas 073100 appears as a very aggressive pathotype on that variety. More generally, criterion $C_{1}$ indicates that 073100 was largely dominant over all other compatible pathotypes on Soissons and on Isengrain and, reciprocally, criterion $C_{2}$ indicates that 073100 was mainly found on these two varieties. This pathotype thus appears as an aggressive specialist. By contrast, according to the same criteria, pathotype 077317 was characterized as a generalist, with no preference for any of the varieties considered.

It then appears that quantitative effects that can be linked to the host quantitative resistance and the pathogen aggressiveness level had a major effect on the leaf rust population structure in France between 1999 and 2008. Such interactions should be taken into account for designing varietal allocation strategies but they are generally not documented. In a recent study, Skelsey et al. (2009) measured aggressiveness traits (Pariaud et al., 2009b) of two Phytophthora infestans isolates on five potato varieties in order to parameterize a spatiotemporal model of potato late blight epidemics. This approach made it possible to obtain specific values for different quantitative parameters underlying the host-pathogen relationship but was restricted, for practical reasons, to a set of isolates and varieties that was not necessarily representative of the field populations. Our approach can be seen as complementary as it did not provide specific values of aggressiveness traits but globally identified the associations between pathotypes and varieties at the landscape scale. Note however that $P$. triticina has a clonal population structure (Goyeau et al., 2007), which makes the establishment of associations between pathotypes and host genotypes easier.

Even though the basic affinity (in terms of our model) between a pathotype and a variety strongly influenced the pathogen population structure, its effect was modulated by that of the other varieties present in the landscape. Indeed, 
the analysis of the link between the pathogen population composition and the varietal frequencies in the cultivated landscape showed that a variety, by increasing the population size of a pathotype, may significantly influence the composition of the pathogen population on other varieties. This effect is quantified in the model by parameter $\beta$ (see Eqn 1a). For example, the variety Caphorn is expected to have a strong influence on the presence of pathotype 106314 on the other varieties. This can be seen in Fig. 5, where the presence of 106314 on Soissons, Apache and Orvantis is mainly accounted for by the frequency of Caphorn in the landscape. The population size of a pathotype on a variety thus resulted from its aggressiveness level on that variety as well as from inoculum produced by other varieties, and the model was able to characterize both effects.

Another major conclusion of our analysis is that the observed resistance level of a variety could be linked to the composition of the pathogen population, which itself depended on the landscape composition (as seen above). The link between the expected disease severity on a variety and the pathotype frequencies is quantified in the model by parameter $b$ (see Eqn 2a). It is usual to observe a resistance breakdown when a major resistance gene is overcome by a new pathotype. In the case of quantitative resistance, a gradual decline is expected when the frequency of aggressive individuals increases in the pathogen population. A comparison of both situations can be found in Mundt et al. (2002).

Some varieties maintained a fairly constant observed level of resistance over the period considered (Fig. 2). For example, the fact that no pathotype presented a marked affinity for Apache or was linked to its observed resistance level is consistent with a high level of quantitative resistance in this variety. The situation was different for Orvantis. This variety was released in 2000 and it exhibited a decreasing level of observed resistance until the end of the period studied (Fig. 2). This decrease could be attributed to pathotype 106314 and, to a lesser extent, 006xxx and 077317. Both 106314 and 006xxx were sensitive to changes in Caphorn's frequency and it seems that the influence of Caphorn on the pathogen population (see Fig. 5c,d,f) contributed to the decline in Orvantis' resistance.

Resistance breakdowns are frequent in crops, but it is less common to observe an increase in the observed resistance level of a variety. This is, however, what happened here for Soissons. The disease scores obtained on Soissons were strongly linked to the frequency of 073100 in the pathogen population (see parameter $b$ in Table 2) and, therefore, the relative decline in 073100 accounts for the increase in Soissons' observed resistance level. After 2002, 073100 was partly replaced by 077317 and 106314 , both of which produce susceptible-type lesions on Soissons (Goyeau et al., 2006) but appear to be much less aggressive than 073100 in the field (Table 2) and, as a result, probably do not cause severe epidemics on that variety. The increase in the freq- uency of 077317 and 106314 on Soissons can be attributed to the influence of other varieties (Figs 5b,c). The decrease in 073100 on Soissons followed the decline in Soissons' frequency after the mid-1990s and resulted from the fact that this pathotype was not compatible with other varieties, except Isengrain. In recent years, Soissons has been gradually rated as more and more resistant by extension services. We were able to establish here that this increased resistance did not result from a global decrease of the virulent population but was linked to the frequency of a single highly aggressive pathotype.

In order to build and estimate the model, it was assumed that the pathotype frequencies were independent between years and that the geographical distribution of the pathotypes was homogeneous at the scale of the country. Given that we worked at the country scale and with data collected at the end of the epidemic season, it is reasonable to assume that the effect of the current year on the pathogen population structure was predominant over the potential effect of previous years. A preliminary exploration of the datasets supported this hypothesis. The assumption of a homogeneous pathotype distribution at the scale of the country was consistent with the population structures described for leaf rust (Goyeau et al., 2006, 2007), and the fact that rust spores are dispersed over large distances (Park et al., 2000). We also assumed that the five main pathotypes considered in the study were pre-existing and homogeneously distributed at the country scale. Theoretical models predict that local structures in the host population could be crucial in the invasion dynamics of pathogens (Keeling, 1999; Park et al., 2001; Débarre et al., 2009). A possible improvement of the analysis would be to test whether weakening the assumption of complete mixing of the population at the national level would alter the results.

Another limit of the study resulted from boundary effects in the datasets. We worked on a sequence of 10 consecutive years, which was long enough to capture major changes in the host and pathogen populations. Nevertheless, the beginning of the period may have been influenced by what happened before 1999. Moreover, the model cannot estimate the parameters for varieties that were introduced too late towards the end of the period as this estimation requires a certain amount of information. In particular, a decrease in the landscape representativeness occurred in 2007 and 2008 because of the rise of a new variety, Sankara, which represented $8-9 \%$ of the wheat landscape those last $2 \mathrm{yr}$. This variety bears the same resistance genes as Caphorn and Orvantis. It is susceptible to pathotype 106314 and high disease score values were recorded on Sankara in the field severity assessments. It is therefore likely that this variety played a role in the multiplication of pathotype 106314, and it is possible that the large influence on 106314 attributed to Caphorn was partly overestimated if the effect of Sankara was confused with that of Caphorn. Based on the 
existing dataset, the specific effect of Sankara cannot yet be properly assessed by the model because the introduction of this variety in the system is too recent.

The approach developed here provided documented situations and information that can be used with landscape epidemiology models for designing variety management strategies. It also made it possible to identify major effects that have to be taken into account in the simulation of large-scale epidemics. Based on parameters $\alpha$ and $\beta$, interaction groups can be defined that account for both qualitative (gene-for-gene) and quantitative host-pathogen interactions in a landscape: pathotype 073100 was clearly related to Isengrain and Soissons; 006xxx was influenced by Caphorn and Orvantis and 106314 by Caphorn; 016206 was influenced by Trémie with a moderate influence of Charger; 077317 appeared as a generalist and was not linked to a specific variety. This pattern can be linked to ecological specialization (Devictor et al., 2010). In that field of research, species specialisation indices are commonly calculated at the landscape scale, for example to understand the impact of human activities on the structure of natural communities (Clavero \& Brotons, 2010). The present study suggests that such specialisation indices could be relevant in plant epidemiology to identify pathotype preferences in a heterogeneous host population and to better understand and predict pathogen population dynamics over the years in cultivated landscapes.

\section{References}

Altieri MA. 1999. The ecological role of biodiversity in agroecosystems. Agriculture Ecosystems \& Environment 74: 19-31.

Barrès B, Halkett F, Dutech C, Andrieux A, Pinon J, Frey P. 2008. Genetic structure of the poplar rust fungus Melampsora larici-populina: evidence for isolation by distance in Europe and recent founder effects overseas. Infection, Genetics \& Evolution 8: 577-587.

Bianchi FJJA, Booij CJH, Tscharntke T. 2006. Sustainable pest regulation in agricultural landscapes: a review on landscape composition, biodiversity and natural pest control. Proceedings of the Royal Society BBiological Sciences 273: 1715-1727.

Bolton MD, Kolmer JA, Garvin DF. 2008. Wheat leaf rust caused by Puccinia triticina. Molecular Plant Pathology 9: 563-575.

Brun H, Chèvre AM, Fitt BDL, Powers S, Besnard AL, Ermel M, Huteau V, Marquer B, Eber F, Renard M et al. 2010. Quantitative resistance increases the durability of qualitative resistance to Leptosphaeria maculans in Brassica napus. New Phytologist 185: 285-299.

Clavero M, Brotons L. 2010. Functional homogenization of bird communities along habitat gradients: accounting for niche multidimensionality. Global Ecology and Biogeography 19: 684-696.

Débarre F, Lenormand T, Gandon S. 2009. Evolutionary epidemiology of drug-resistance in space. PLoS Computational Biology 5: e1000337.

Devictor V, Clavel J, Julliard R, Lavergne S, Mouillot D, Thuiller W, Venail P, Villéger S, Mouquet N. 2010. Defining and measuring ecological specialization. Journal of Applied Ecology 47: 15-25.

Flor HH. 1971. Current status of the gene-for-gene concept. Annual Review of Phytopathology 9: 275-296.

Garrett KA, Zúñiga LN, Roncal E, Forbes GA, Mundt CC, Su Z, Nelson RJ. 2009. Intraspecific functional diversity in hosts and its effect on disease risk across a climatic gradient. Ecological Applications 19: $1868-1883$.

Gelman A, Carlin JB, Stern HS, Rubin DB. 2004. Bayesian data analysis, 2nd edn. New York, NY, USA: Chapman and Hall/CRC.

Gilligan CA. 2008. Sustainable agriculture and plant diseases: an epidemiological perspective. Philosophical Transactions of the Royal Society B-Biological Sciences 363: 741-759.

Gilmour J. 1973. Octal notation for designating physiologic races of plant pathogens. Nature 242: 620.

Goyeau H, Halkett F, Zapater MF, Carlier J, Lannou C. 2007. Clonality and host selection in the wheat pathogenic fungus Puccinia triticina. Fungal Genetics and Biology 44: 474-483.

Goyeau H, Park R, Schaeffer B, Lannou C. 2006. Distribution of pathotypes with regard to host cultivars in French wheat leaf rust populations. Phytopathology 96: 264-273.

Hanski I. 1998. Metapopulation dynamics. Nature 396: 41-49.

Hovmøller MS, Munk L, Østergård H. 1993. Observed and predicted changes in virulence gene-frequencies at 11 loci in a local barley powdery mildew population. Phytopathology 83: 253-260.

Keeling MJ. 1999. The effects of local spatial structure on epidemiological invasions. Proceedings of the Royal Society B-Biological Sciences 266: 859-867.

Keesing F, Holt RD, Ostfeld RS. 2006. Effects of species diversity on disease risk. Ecology Letters 9: 485-498.

Kolmer JA. 2002. Virulence phenotypes of Puccinia triticina in the South Atlantic States in 1999. Plant Disease 86: 288-291.

Marcel TC, Gorguet B, Ta MT, Kohutova Z, Vels A, Niks RE. 2008. Isolate specificity of quantitative trait loci for partial resistance of barley to Puccinia hordei confirmed in mapping populations and near-isogenic lines. New Phytologist 177: 743-755.

Margosian ML, Garrett KA, Shawm Hutchinson JM, With KA. 2009. Connectivity of the American agricultural landscape: assessing the national risk of crop pest and disease spread. BioScience 59: 141-151.

Miller JS, Johnson DA, Hamm PB. 1998. Aggressiveness of isolates of Phytophthora infestans from the Columbia basin of Washington and Oregon. Phytopathology 88: 190-197.

Mundt CC. 2002. Use of multiline cultivars and cultivar mixtures for disease management. Annual Review of Phytopathology 40: 381-410.

Mundt CC, Brophy LS. 1988. Influence of number of host genotype units on the effectiveness of host mixtures for disease control: a modeling approach. Phytopathology 78: 1087-1094.

Mundt CC, Cowger C, Garrett KA. 2002. Relevance of integrated disease management to resistance durability. Euphytica 124: 245-252.

Oerke EC, Dehne HW. 2004. Safeguarding production - losses in major crops and the role of crop protection. Crop Protection 23: 275-285.

ONIGC. 2008. Répartition des variétés - récoltes 1999-2008 (blé tendre, orge, blé dur, triticale). Technical Report: Les Cahiers de l'ONIGC. Paris, France: France Agrimer.

Pariaud B, Ravigne V, Halkett F, Goyeau H, Carlier J, Lannou C. 2009a. Aggressiveness and its role in the adaptation of plant pathogens. Plant Pathology 58: 409-424.

Pariaud B, Robert C, Goyeau H, Lannou C. 2009b. Aggressiveness components and adaptation to a host cultivar in wheat leaf rust. Phytopathology 99: 869-878.

Park AW, Gubbins S, Gilligan CA. 2001. Invasion and persistence of plant parasites in a spatially structured host population. Oikos 94 : 162-174.

Park RF, Jahoor A, Felsenstein FG. 2000. Population structure of Puccinia recondite in western Europe during 1995, as assessed by variability in pathogenicity and molecular markers. Journal of Phytopathology 148: 169-179.

Parnell S, van den Bosch F, Gilligan CA. 2006. Large-scale fungicide spray heterogeneity and the regional spread of resistant pathogen strains. Phytopathology 96: 549-555. 
Pautasso M, Holdenrieder O, Stenlid J. 2005. Susceptibility to fungal pathogens of forests differing in tree diversity. Ecological Studies 176: 263-289.

Plantegenest M, Le May C, Fabre F. 2007. Landscape epidemiology of plant diseases. Journal of the Royal Society Interface 4: 963-972.

Plummer M. 2010. JAGS Version 2.1.0 user manual. Technical Report. [WWW document]. URL http://www-fis.iarc.fr/-martyn/software/jags/ [accessed 11 March 2011].

Rouxel T, Penaud A, Pinochet X, Brun H, Gout L, Delourme R, Schmit J, Balesdent MH. 2003. A 10-year survey of populations of Leptosphaeria maculans in France indicates a rapid adaptation towards the $\mathrm{rlm} 1$ resistance gene of oilseed rape. European Journal of Plant Pathology 109: 871-881.

Samborski DJ. 1985. Wheat leaf rust. In: Roelfs AP, Bushnall WR, eds. The cereal rusts 2. Orlando, FL, USA: Academic Press, 39-52.

Skelsey P, Kessel GJT, Rossing WAH, van der Werf W. 2009.

Parameterization and evaluation of a spatiotemporal model of the potato late blight pathosystem. Phytopathology 99: 290-300.

Stukenbrock EH, McDonald BA. 2008. The origins of plant pathogens in agro-ecosystems. Annual Review of Phytopathology 46: 75-100.

Thrall PH, Burdon JJ. 2003. Evolution of virulence in a plant hostpathogen metapopulation. Science 299: 1735-1737.

Tildesley MJ, House TA, Bruhn MC, Curry RJ, O’Neil M, Allpress JLE, Smith G, Keeling MJ. 2010. Impact of spatial clustering on disease transmission and optimal control. Proceedings of the National Academy of Sciences, USA 107: 1041-1046.

Wolfe MS, Schwarzbach E. 1978. Patterns of race changes in powdery mildews. Annual Reviews of Phytopathology 16: 159-180.

Zadoks JC, Kampmeijer P. 1977. The role of crop populations and their deployment, illustrated by means of a simulator, Epimul 76. Annals of the New York Academy of Sciences 287: 164-190.

Zhu Y, Chen H, Fan J, Wang Y, Li Y, Chen J, Fan JX, Yang S, Hu L, Leung $\mathrm{H}$ et al. 2000. Genetic diversity and disease control in rice. Nature 406: 718-722.

\section{Supporting Information}

Additional supporting information may be found in the online version of this article.

Fig. S1 Posterior densities of parameter $\alpha$.

Fig. S2 Posterior densities of parameter $\beta$.

Fig. S3 Posterior densities of parameter $b$.

Fig. S4 Posterior densities of parameters $a^{0}, a^{1}$ and $a^{2}$.

Notes S1 Technical information related to the model construction.

Table S1 Frequencies of the studied varieties from 1999 to 2008 in France

Table S2 Number of collected isolates on each variety

Table S3 Number of collected isolates for each pathotype

Please note: Wiley-Blackwell are not responsible for the content or functionality of any supporting information supplied by the authors. Any queries (other than missing material) should be directed to the New Phytologist Central Office. 